\title{
Apresentação dos Trabalhos de Conclusão de Curso (TCC) de 2002 a 2008 - produção científica dos alunos do curso de graduação em enfermagem da FMABC
}

$\mathrm{O}$

grande desafio da disciplina Estudos Metodológicos, oferecida no curso de graduação em enfermagem da Faculdade de Medicina do ABC (FMABC), não é ensinar sobre o procedimento metodológico, mas "persuadir" o aluno a acreditar que pode fazer pesquisa e por um momento ser, portanto, um pesquisador que produz conhecimento. Tal aspiração não é uma utopia, dado que Aristóteles já afirmava que "todos os homens têm, por natureza, o desejo de conhecer" (Chauí, $\mathrm{M}^{1}$ ); e o conhecimento é a matéria prima para aquele que deseja produzir um novo saber.

Deste modo, este "amor pela sabedoria” (Chauí, $\mathrm{M}^{1}$ ), origem da palavra grega filosofia (philo $=$ amizade e sophia $=$ sabedoria), tem sido o guia para os orientadores trabalharem com seus orientandos.

A certeza das limitações para atingir tais objetivos está presente, porém, é positiva na medida em que se torna motivo do corpo docentediscente para enfrentar este desafio. Humberto $\mathrm{Eco}^{2}$ referiu que “elaborar uma tese é como exercitar a memória”, deste modo, o desafio é também constante.

O referencial adotado para a estratégia metodológica é o Manual de Investigação em Ciências Sociais, de Quivy e Campenhoudt”; uma estratégia na qual o aluno mediante leituras, estudos dirigidos e complementação teórica dos docentes, constrói seu projeto no decorrer da disciplina e com a participação da classe e orientador. Importante destacar que esta metodologia de ensino foi desenvolvida na Faculdade de Saúde Pública da Universidade de São Paulo, pela Professora Doutora Augusta Thereza de Alvarenga e foi adaptada por este curso da FMABC. Enfatizando-se que neste processo o aluno realiza os três atos do procedimento metodológico: a ruptura, a construção e a verificação.

Para atingir estes processos, a disciplina de estudos metodológicos consiste, inclusive, em oferecer subsídios teóricos a fim de que o aluno de graduação em enfermagem tenha capacidade de elaborar seu trabalho de conclusão de curso (TCC) sobre um tema relevante na sua área. Dado que este trabalho acadêmico deva ser elaborado com metodologias de trabalho científico, o primeiro currículo do curso oferecia duas disciplinas para subsidiar o graduando. A metodologia da pesqui- sa, ministrada na segunda série, que oferece os conceitos gerais da teoria do conhecimento e inicia o aluno como "futuro pesquisador" e na quarta série, estudos metodológicos, na qual consiste em desenvolver sua pesquisa.

Com a reestruturação curricular, em 2006, o currículo foi revisado e dentre as alterações, criou-se outra disciplina e distintas foram readequadas para melhor subsidiar o aluno; na primeira série, iniciação científica, metodologia da pesquisa (segunda série), estudos metodológicos I (terceira série) e estudos metodológicos II (quarta série). Esta reestruturação tem permitido que o aluno possa iniciar seu TCC no terceiro ano e, deste modo, produzir um trabalho com um tempo, suporte metodológico da disciplina e orientador maiores.

Destaca-se que, desde a primeira turma, os alunos desenvolvem seus trabalhos com o apoio de um orientador e ao final são submetidos a uma banca avaliadora para o último parecer. A defesa e apresentação limitavam-se, para as primeiras turmas, à própria classe. Contudo, desde 2005, optou-se por ampliar esta atividade e transformá-lo em um evento científico, possibilitando a divulgação destes trabalhos em outras esferas da FMABC, serviços de saúde e familiares dos alunos.

Outro aparte diz respeito às modalidades diversificadas, com realização de pesquisas quantitativas, qualitativas, estudos de revisão e de casos. Além dos trabalhos que se destacaram e foram divulgados com o auxílio dos orientadores, tais como publicação em periódicos e especialmente em eventos científicos, como em congressos conceituados. Soma-se a isto a contribuição que o curso da FMABC vem dando para o progresso da enfermagem.

Assim, esta disciplina procura utilizar e manter o processo científico de maneira sábia e responsável, utilizando a definição de Gaston Bachelard como fio condutor, na qual ele deve ser "um ato científico conquistado sobre os preconceitos, construído pela razão e verificado nos fatos" (Chauí, $\mathrm{M}^{1}$ ). Este compromisso se evidenciou em diversos trabalhos realizados, os quais sobrepuseram o cumprimento acadêmico para obtenção do título da graduação em enfermagem, pois puderam ser divulgados.

\footnotetext{
Chauí, M. Convite à filosofia. 8ªed. Ed.Ática; 1997.

2 Eco, H. Como se faz uma tese. Ed. Perspectiva;SP.14ª ed.1977.

${ }^{3}$ Quivy, R. Campenhoudt, LV. Manual de investigação em ciências sociais. Gradiva. 3ª Ed.Portugal; 2003.
} 
Logo, nossa conquista nestes dez anos de existência na FMABC pode ser verificada a seguir pela apresentação do Quadro 1, que demonstra os temas abordados das produções científicas realizadas pelos alunos deste curso. A seguir, apresentam-se os resumos estruturados da maioria dos TCCs descritos nos quadros, possibilitando conhecer a temática desenvolvida.

É importante enfatizar que esta produção científica dos alunos do curso de graduação de enfermagem foi realizada em parceria com seus orientadores, atores essenciais nesta construção e que merecem um agradecimento especial.

Rosangela Filipini

Disciplina de Estudos Metodológicos do Curso de Enfermagem da Faculdade de Medicina do ABC (FMABC), Santo André (SP), Brasil

Quadro 1 - Relação de trabalhos de conclusão de curso de Graduação em Enfermagem da FMABC de 2002 a 2008

\begin{tabular}{|c|c|c|c|c|c|}
\hline TCC & ANO & TíTULO & ALUNO & ORIENTADOR & RESUMO \\
\hline 1 & 2002 & 0 papel do enfermeiro no aleitamento materno & Ana Carolina Guimarães & Sandra T. Amarante & 001 \\
\hline 2 & 2002 & $\begin{array}{l}\text { Ações de enfermagem na assistência ao pré-natal no serviço } \\
\text { público- estudo de revisão. }\end{array}$ & Bete Queila R. de S. Camilles & Rosangela Filipini & \\
\hline 3 & 2002 & $\begin{array}{l}\text { Parto Humanizado - uma ação de saúde realizada pra a prevenção } \\
\text { dos riscos do parto cesárea. Uma revisão bibliográfica. }\end{array}$ & Camila Sanches de Souza & Rosangela Filipini & \\
\hline 4 & 2002 & Programa de internação domiciliar & Carla R. Yukie Kobayashi & Cecília Melo & \\
\hline 5 & 2002 & $\begin{array}{l}\text { Perfil epidemiológico do paciente atendido no serviço de cardiologia } \\
\text { da FMABC- elaboração do protocolo de consulta de enfermagem }\end{array}$ & Cintia Capilla & Veruska Hernandes & \\
\hline 6 & 2002 & $\begin{array}{l}\text { Atuação do enfermeiro em um serviço especializado a gestantes } \\
\text { de alto risco. }\end{array}$ & Elaine Dilourdes de Souza & Sônia R.G. Lara & \\
\hline 7 & 2002 & $\begin{array}{l}\text { Mapeamento do serviço de emergência na região do } \mathrm{ABC} \text { quanto } \\
\text { aos aspectos físicos, materiais e humanos. }\end{array}$ & Erica Chagas Araújo & Ana Maria Fiorano & 002 \\
\hline 8 & 2002 & $\begin{array}{l}\text { Relacionamento terapêutico: a importância e aplicabilidade na } \\
\text { prática de enfermagem }\end{array}$ & Heloisa Morishita & M. do Perpétuo Socorro de Nóbrega & \\
\hline 9 & 2002 & $\begin{array}{l}\text { Conhecimento teórico dos profissionais de enfermagem de um } \\
\text { Hospital privado sobre o cuidado geronto-geriátrico }\end{array}$ & Kelin Cristina Frank & Ana Paula Guarnieri & \\
\hline 10 & 2002 & $\begin{array}{l}\text { Interdisciplinaridade no processo de trabalho em saúde instituci- } \\
\text { onalizada }\end{array}$ & Luciane Morelis & Sandra T. Amarante & \\
\hline 11 & 2002 & Perfil do cliente idoso num Hospital privado do $A B C$. & Lyana P. R. Scotto Di Santillo & Ana Paula Guarnieri & \\
\hline 12 & 2002 & $\begin{array}{l}\text { Análise da implantação do Programa de saúde da Família num } \\
\text { município do ABC. }\end{array}$ & Miriam de Menezes Cunha & Loide Corina Chaves & \\
\hline 13 & 2002 & Aborto na adolescência: um problema de saúde pública & Olivia Marie Gaube & Sônia R.G. Lara & \\
\hline 14 & 2002 & $\begin{array}{l}\text { Utilização do Clopidogrel na prevenção de eventos cardíacos } \\
\text { isquêmicos por Angioplastia Transluminal Percutânea. }\end{array}$ & Rebecca Pessoto Pires & Ana Maria Fiorano & 003 \\
\hline 15 & 2002 & $\begin{array}{l}\text { Brinquedo terapêutico - Humanizando a assistência de enfermagem } \\
\text { à criança hospitalizada.Uma revisão bibliográfica }\end{array}$ & Regiane Gimenez Mordente & Rosangela Filipini & \\
\hline 16 & 2002 & $\begin{array}{l}\text { Caracterização das percepções dos clientes idosos e/ou familiares } \\
\text { sobre a assistência de enfermagem prestada num hospital do ABC. }\end{array}$ & Regiane Polizer & Ana Paula Guarnieri & \\
\hline 17 & 2002 & $\begin{array}{l}\text { Infecção do trato urinário em pacientes com cateterismo vesical de } \\
\text { demora atendidos em dois hospitais públicos as região do } A B C \text {. }\end{array}$ & Roberta do Nascimento Barbosa & Loide Corina Chaves & 004 \\
\hline 18 & 2002 & Os sentimentos do paciente oncológico. & Sabrina de Almeida & Simone de Oliveira Camillo & \\
\hline 19 & 2002 & Violência contra o idoso no Brasil. & Simone dos Santos Fialho & M. do Perpétuo Socorro de Nóbrega & \\
\hline 20 & 2002 & $\begin{array}{l}\text { Perfil do enfermeiro que atua junto a dependentes químicos em } \\
\text { serviços de saúde especializados. }\end{array}$ & Tathiany de Lima Nemitz & M. do Perpétuo Socorro de Nóbrega & \\
\hline 21 & 2002 & $\begin{array}{l}\text { Liderança da equipe de enfermagem: caracterização do conheci- } \\
\text { mento e estratégias pra a atuação do enfermeiro }\end{array}$ & Vivian Cristina Oyama & Sandra T. Amarante & \\
\hline 22 & 2002 & Humanização em Unidade de Terapia Intensiva & Vivian Zogheib & Ana Maria Fiorano & \\
\hline 23 & 2003 & $\begin{array}{l}\text { Atuação do enfermeiro no acompanhamento do paciente portador } \\
\text { de marcapasso. }\end{array}$ & Alessandra da Conceição Chaves & Ana Maria Fiorano & \\
\hline 24 & 2003 & $\begin{array}{l}\text { A influência do lazer na qualidade de vida de idosos institucionaliza- } \\
\text { dos portadores de incapacidade física funcional. }\end{array}$ & Aline Cristina Monteiro & Ana Paula Guarnieri & \\
\hline 25 & 2003 & $\begin{array}{l}\text { Perfil do funcionário acompanhante de idosos de uma Instituição } \\
\text { asilar. }\end{array}$ & Ana Maria B. Perrone & Ana Paula Guarnieri & \\
\hline 26 & 2003 & $\begin{array}{l}\text { Humanização na unidade de terapia intensiva: sentimentos dos } \\
\text { familiares de pacientes sob ventilação mecânica. }\end{array}$ & Camila Aleixo Camargo & Ana Maria Fiorano & 005 \\
\hline 27 & 2003 & $\begin{array}{l}\text { Cirurgia Bariátrica como tratamento da obesidade mórbida: Moti- } \\
\text { vação do paciente para realizá-la. }\end{array}$ & Cleide A. S. Gonçalves & M. do Perpétuo Socorro de Nóbrega & \\
\hline 28 & 2003 & Influência na orientação do enfermeiro na aderência ao tratamento. & Cristiane Leimi Oshiro & Ana Paula Guarnieri & \\
\hline 29 & 2003 & $\begin{array}{l}\text { A primeira experiência em campo de estágio hospitalar: sentimen- } \\
\text { tos dos acadêmicos do segundo ano do curso de Enfermagem. }\end{array}$ & Fernanda do Vale Soares & Simone de Oliveira Camillo & 006 \\
\hline 30 & 2003 & Qualidade de vida dos acadêmicos trabalhadores de enfermagem. & Gabriela P. de Assis & Sandra T. Amarante & 007 \\
\hline
\end{tabular}




\begin{tabular}{|c|c|c|c|c|c|}
\hline TCC & ANO & TÍTULO & ALUNO & ORIENTADOR & RESUMO \\
\hline 31 & 2003 & $\begin{array}{l}\text { A influência do método canguru no ganho de peso do recém- } \\
\text { nascido de baixo peso. }\end{array}$ & Glauce Lopes Castello & Simone Garcia Lopes & \\
\hline 32 & 2003 & Motivos da Escolha do tipo de Parto: Normal ou Casaria. & Joyce Ellero Fernandes & Sônia R.G. Lara & \\
\hline 33 & 2003 & $\begin{array}{l}\text { Riscos para o câncer cérvico-uterino em mulheres atendidas no } \\
\text { projeto GRAM da FMABC. }\end{array}$ & Juliana Alves Ferreira & Rosangela Filipini & 008 \\
\hline 34 & 2003 & Frequência da higiene oral em pacientes internados. & Márcia Honda & Maria Elisa G. R. Ramos & \\
\hline 35 & 2003 & $\begin{array}{l}\text { Critérios estabelecidos no uso de roupa privativa em ambiente } \\
\text { cirúrgico. }\end{array}$ & Maria Lúcia Mantovane & Simone Garcia Lopes & 009 \\
\hline 36 & 2003 & Cuidados paliativos para o alívio da dor em pacientes oncológicos. & Marisa Sabatini Lima & Alexandra Noemi Silva & \\
\hline 37 & 2003 & $\begin{array}{l}\text { Tratamento medicamentoso: o significado para pacientes com } \\
\text { esquizofrenia. }\end{array}$ & Paloma C. Monteiro Peiniger & Simone de Oliveira Camillo & 010 \\
\hline 38 & 2003 & $\begin{array}{l}\text { Atuação do enfermeiro obstétrico no puerpério imediato. Tendên- } \\
\text { cias atuais. }\end{array}$ & Patrícia Borges Germani & Sônia R.G. Lara & \\
\hline 39 & 2003 & $\begin{array}{l}\text { Recursos utilizados para o tratamento da dor em pessoas com } 60 \\
\text { anos ou mais residentes em Capuava. }\end{array}$ & Patrícia de Pádua Freitas & Ana Paula Guarnieri & \\
\hline 40 & 2003 & $\begin{array}{l}\text { Tendências atuais da assistência de Enfermagem no PO de } \\
\text { transplante de coração, tendo em vista a imunossupressão: uma } \\
\text { revisão da literatura. }\end{array}$ & Regiane Alexandre Vidotti & Ana Maria Fiorano & \\
\hline 41 & 2003 & $\begin{array}{l}\text { Refluxo gastro-esofágico e cuidados de enfermagem. Estudo de } \\
\text { revisão. }\end{array}$ & Symoni Rodrigues Silveira & Isabel C. Fernandes & \\
\hline 42 & 2003 & $\begin{array}{l}\text { Atuação do enfermeiro na rede básica de saúde do } \mathrm{ABC} \text { na as- } \\
\text { sistência ao indivíduo cardiopata }\end{array}$ & Thaís A. Bernardino & Loide Corina Chaves & \\
\hline 43 & 2003 & $\begin{array}{l}\text { Benefícios da equoterapia na reabilitação de crianças com ativi- } \\
\text { dades de vida diária afetadas }\end{array}$ & Thais Y. de Campos & Loide Corina Chaves & \\
\hline 44 & 2003 & Infecção pulmonar por Hantavírus. Estudo de revisão. & Vanessa Fachini & Loide Corina Chaves & \\
\hline 45 & 2003 & $\begin{array}{l}\text { Percepções sobre a fase do climático por mulheres em acompanha- } \\
\text { mento ginecológico. }\end{array}$ & Vanessa Gentil & M. do Perpétuo Socorro de Nóbrega & \\
\hline 46 & 2004 & $\begin{array}{l}0 \text { olhar do aluno: revelando o curso de graduação de Enfermagem } \\
\text { após o estágio de Saúde Mental }\end{array}$ & Alan Jefferson do Nascimento & Simone de Oliveira Camillo & 011 \\
\hline 47 & 2004 & $\begin{array}{l}\text { As dificuldades do enfermeiro do centro cirúrgico em exercer sua } \\
\text { prática de trabalho }\end{array}$ & Ana Carolina A. Betini Datilio & Sandra T. Amarante & \\
\hline 48 & 2004 & $\begin{array}{l}\text { Úlcera de Pressão: Atualização acerca da sua prevenção e } \\
\text { tratamento. }\end{array}$ & André Tembikoski Junior & Ana Maria Fiorano & \\
\hline 49 & 2004 & $\begin{array}{l}\text { Perfil socioeconômico e tipo de assistência pré-natal de recém } \\
\text { nascidos de baixo peso. }\end{array}$ & Carina Pescuma Prado & Rosangela Filipini & \\
\hline 50 & 2004 & $\begin{array}{l}\text { Exame Físico de Enfermagem: Opinião dos alunos de Graduação } \\
\text { sobre sua execução. }\end{array}$ & Célia Regina Ferreira da Silva & Sandra T. Amarante & \\
\hline 51 & 2004 & $\begin{array}{l}\text { A importância da atuação do Enfermeiro na Tomografia Computa- } \\
\text { dorizada. }\end{array}$ & Claudia Almendra & Sandra T. Amarante & \\
\hline 52 & 2004 & HIV/AIDS na gravidez: ações de saúde no município de SBC. & Daniela Demarchi & Ana Paula Guarnieri & \\
\hline 53 & 2004 & Fatores determinantes para Depressão infantil & Daniela Ribeiro Amorim & Isabel C. Fernandes & \\
\hline 54 & 2004 & Auto-estima em mulheres usuárias de álcool e drogas & Giovana Mara Manzari Paschoal & M. do Perpétuo Socorro de Nóbrega & 012 \\
\hline 55 & 2004 & $\begin{array}{l}\text { Dificuldades encontradas pelas gestantes atendidas em uma } \\
\text { instituição pública estadual para a realização do Pré-natal. }\end{array}$ & Grace de Agostini Pacheco & Isabel C. Fernandes & \\
\hline 56 & 2004 & Depressão em Graduandos de Enfermagem & Igor Garro Moreira Barbosa & Simone de Oliveira Camillo & 013 \\
\hline 57 & 2004 & $\begin{array}{l}\text { O papel do Enfermeiro na promoção da saúde do Idoso no contexto } \\
\text { do Sistema Único de Saúde (SUS) }\end{array}$ & Juliana Galantim & Ana Paula Guarnieri & \\
\hline 58 & 2004 & Percepções das mães com relação ao médico Mãe-Canguru. & Kássia Almeida Silvério & Sônia R.G. Lara & 014 \\
\hline 59 & 2004 & $\begin{array}{l}\text { Caracterização de crianças portadoras de câncer atendidas em } \\
\text { ambulatório quanto desenvolvimento neuropsicomotor. }\end{array}$ & Maria Isabel Malatesta & Rosangela Filipini & 015 \\
\hline 60 & 2004 & A gestação na adolescência e seus aspectos emocionais. & Nina Márcia Pusch & Ana Maria Fiorano & 016 \\
\hline 61 & 2004 & $\begin{array}{l}\text { Compreensão dos pacientes que fazem Hemodiálise sobre } 0 \\
\text { transplante renal. }\end{array}$ & Raquel Marçon Satore & Alexandra Noemi Silva & \\
\hline 62 & 2004 & $\begin{array}{l}\text { Cuidados de Enfermagem na administração de Quimioterápicos e } \\
\text { seus efeitos colaterais. }\end{array}$ & Renata Delgado Lalli & Maria Elisa G. R. Ramos & 017 \\
\hline 63 & 2004 & $\begin{array}{l}\text { Amamentação: Visão da Puérpera frente as orientações fornecidas } \\
\text { pela equipe de enfermagem. }\end{array}$ & Renata Higa & Sônia A. Gonçalves & 018 \\
\hline 64 & 2004 & $\begin{array}{l}\text { Pacientes atendidos no "Projeto Bem Viver" de um Centro de } \\
\text { saúde escola do Grande ABC: Diagnostico de Enfermagem mais } \\
\text { freqüentes. }\end{array}$ & Silvana Loddi & Loide Corina Chaves & 019 \\
\hline 65 & 2004 & Presença de Crise de vida em graduandos em Enfermagem. & Simone Meneghello & M. do Perpétuo Socorro de Nóbrega & 020 \\
\hline 66 & 2004 & Prováveis causas da não adesão dos hipertensos ao tratamento. & Tânia Raquel C. C.Moreira & Alexandra Noemi Silva & \\
\hline 67 & 2004 & Parto Natural: Uma mudança de Paradigma. & Thatiana Miller F. Ruaro & Sônia R.G. Lara & 021 \\
\hline
\end{tabular}




\begin{tabular}{|c|c|c|c|c|c|}
\hline TCC & ANO & TíTULO & ALUNO & ORIENTADOR & RESUMO \\
\hline 68 & 2005 & $\begin{array}{l}\text { Relacionamento enfermeiro, paciente e família: fatores comporta- } \\
\text { mentais associados à assistência. }\end{array}$ & Amanda Batista de Siqueira & Rosangela Filipini & 022 \\
\hline 69 & 2005 & $\begin{array}{l}\text { Gerenciamento do tempo pela equipe de Enfermagem em uma } \\
\text { Unidade de Integração Pediátrica. }\end{array}$ & Camila Silva Niero & Sandra T. Amarante & \\
\hline 70 & 2005 & $\begin{array}{l}\text { Atuação do Enfermeiro na Assistência Prestada ao Paciente com } \\
\text { Síndrome Sundown. }\end{array}$ & Cilene B. N. Lourenço & Maria Elisa G. R. Ramos & 023 \\
\hline 71 & 2005 & $\begin{array}{l}\text { Projeto Bem Viver: Avaliando uma proposta de Intervenção de } \\
\text { Enfermagem gerontológica na comunidade. }\end{array}$ & Danívea B. Poltronieri & Ana Paula Guarnieri & 024 \\
\hline 72 & 2005 & $\begin{array}{l}\text { Percepção de Enfermeiros mediante a aderência de pacientes em } \\
\text { CAPD e suas intervençôes para inclusão ao método diálitico. }\end{array}$ & Débora Abrão Calderan & Ana Maria Fiorano & \\
\hline 73 & 2005 & $\begin{array}{l}\text { Venda de bebida alcoólica e violência: } 0 \text { que pensam os donos de } \\
\text { bares. }\end{array}$ & Diego Franchino & M. do Perpétuo Socorro de Nóbrega & 025 \\
\hline 74 & 2005 & $\begin{array}{l}\text { Perfil da população idosa em uma Unidade Básica de Saúde do } \\
\text { Município de Santo André. }\end{array}$ & Edina Ferreira Panaino & Ana Paula Guarnieri & \\
\hline 75 & 2005 & $\begin{array}{l}0 \text { olhar do aluno do Curso de Graduação de Enfermagem em } \\
\text { Relação aos pacientes com Transtorno mentais Após o Estágio de } \\
\text { Psiquiatria. }\end{array}$ & Flaviane Kesia R. Alonso & Simone de Oliveira Camillo & 026 \\
\hline 76 & 2005 & Consumo de Drogas Lícitas e llícitas por enfermeiros. & Ivani C. Santos Costa & Rosangela Filipini & \\
\hline 77 & 2005 & $\begin{array}{l}\text { Análise do procedimento de limpeza de sala de operação em } \\
\text { Hospitais de Ensino Vinculados Á Fundação ABC. }\end{array}$ & Jaqueline Poltronieri & Simone Garcia Lopes & 027 \\
\hline 78 & 2005 & Convivendo e Enfrentando o Câncer de Mama. & Juliana Moreira & Simone de Oliveira Camillo & 028 \\
\hline 79 & 2005 & Organização do trabalho na equipe de saúde da família. & Luana A. Beltramo & Sandra T. Amarante & 029 \\
\hline 80 & 2005 & Fatores que dificultam a redução de peso entre idosos. & Luiza Maria S. de Camargo & Ana Paula Guarnieri & \\
\hline 81 & 2005 & Gestão Humanizada na Equipe de Enfermagem. & Maria do Carmo N. Costa & $\begin{array}{l}\text { Adozinda de F. Marques Henriques } \\
\text { da Silveira }\end{array}$ & \\
\hline 82 & 2005 & Uso do adesivo de psicotrópicos em idosos. & Marina Teixeira Santos & Maria Elisa G. R. Ramos & \\
\hline 83 & 2005 & $\begin{array}{l}\text { Condutas de enfermagem desenvolvida na reabilitação plantar de } \\
\text { idosos em uma equipe multiprossional: recriando o cuidado }\end{array}$ & Natalia Zerbinatti Salvador & Ana Paula Guarnieri & \\
\hline 84 & 2005 & $\begin{array}{l}\text { Infecção pelo HIV: conhecimento apresentado pela equipe de } \\
\text { higiene de um hospital público da Região do ABC. }\end{array}$ & Rosemary F. C. Lourencetti & Loide Corina Chaves & 030 \\
\hline 85 & 2005 & $\begin{array}{l}\text { Causas mais comum de acidentes na infância em crianças de } 0 \text { a } \\
14 \text { anos. }\end{array}$ & Telma Magali M. Barros & Isabel C. Fernandes & \\
\hline 86 & 2005 & Assistência de Enfermagem á Gestante com DHEG. & Úrsula Dias Medeiros & Sônia R.G. Lara & \\
\hline 87 & 2005 & A percepção da sexualidade na mulher idosa atual. & Vanessa Morrone de Castilho & $\begin{array}{l}\text { Simone Garcia Lopes e Ana Paula } \\
\text { Guarnieri }\end{array}$ & 031 \\
\hline 88 & 2005 & 0 conhecimento do Idoso sobre DST e AIDS. & Wanderléia Cristina dos Santos & Loide Corina Chaves & 032 \\
\hline 89 & 2006 & $\begin{array}{l}\text { Humanização do cuidado: conhecimento e aplicabilidade de profis- } \\
\text { sionais da saúde em ambiente intra-hospitalar. }\end{array}$ & Aine Emanuelle Stanziani & Simone Garcia Lopes & 033 \\
\hline 90 & 2006 & $\begin{array}{l}\text { Centro cirúrgico ambulatorial: como construir e/ou adaptar este } \\
\text { serviço. }\end{array}$ & Aline C. Foroni Siqueira & Simone Garcia Lopes & 034 \\
\hline 91 & 2006 & Implantação da SAE em sala de emergência & Aline Soria Fernandes & Ana Maria Fiorano & \\
\hline 92 & 2006 & Dor no Recém-nascido: Percepções do Enfermeiro. & Ana Carolina Marchiore & Sônia A. Gonçalves & 035 \\
\hline 93 & 2006 & $\begin{array}{l}\text { A doença hipertensiva específica da gestação na terapia intensiva: } \\
\text { o preparo do enfermeiro intensivista. }\end{array}$ & Ana Paula dos Santos Paschoal & Érica C. Araújo & 036 \\
\hline 94 & 2006 & $\begin{array}{l}\text { Mapeamento do Serviço de Emergência na Região do Grande ABC: } \\
\text { Recursos Físicos, Materiais e Humanos em } 2006 .\end{array}$ & Andrea lartelli & Ana Maria Fiorano & 037 \\
\hline 95 & 2006 & $\begin{array}{l}\text { Identificação da aderência de ácido fólico nos três primeiros meses } \\
\text { de gestação. }\end{array}$ & Andrea Madalena Mendes & Sônia R.G. Lara & 038 \\
\hline 96 & 2006 & $\begin{array}{l}\text { Conhecimentos e adesão dos profissionais de enfermagem quanto } \\
\text { aos resíduos de serviços de saúde. }\end{array}$ & Bruna de Souza de Azevedo & Isabel C. Fernandes & \\
\hline 97 & 2006 & Atuação do enfermeiro na promoção da saúde do idoso. & Camila Cabral Bruno & Ana Paula Guarnieri & \\
\hline 98 & 2006 & $\begin{array}{l}\text { Perfil do paciente que realiza hemodiálise em um centro de saúde } \\
\text { escola em Santo André. }\end{array}$ & Carolina Palopoli & Ana Maria Fiorano & \\
\hline 99 & 2006 & $\begin{array}{l}\text { Comunicação humanizada entre profissional de saúde e paciente no } \\
\text { centro cirúrgico: a importância do uso do nome }\end{array}$ & Caroline Nitta Rodrigues & Márcia Tamosauskas & 039 \\
\hline 100 & 2006 & $\begin{array}{l}\text { Proposta de implantação da Neonatal Infant Pain Scale (NIPS) na } \\
\text { Unidade de Terapia Intensiva Neonatal. }\end{array}$ & Cibele Sachetto & Sônia A. Gonçalves & 040 \\
\hline 101 & 2006 & $\begin{array}{l}\text { Relação entre condições socioeconômicas e conhecimentos sobre } \\
\text { método preventivo }\end{array}$ & Cláudia Marina dos Santos Cecilio & Lourdes da Conceição Tavares & \\
\hline 102 & 2006 & $\begin{array}{l}\text { O trabalho como fator de risco ou de proteção para o consumo de } \\
\text { álcool e drogas em homens da FMABC. }\end{array}$ & Cristiane Ortiz Flora & M. do Perpétuo Socorro de Nóbrega & \\
\hline 103 & 2006 & $\begin{array}{l}\text { Emoções vivenciadas pelos enfermeiros que atuam na unidade de } \\
\text { emergência. }\end{array}$ & Danielle A. Xavier de Assis & Ana Maria Fiorano & \\
\hline 104 & 2006 & $\begin{array}{l}\text { Aceitação do aleitamento materno em recém-nascidos pré-termos } \\
\text { que foram entubados. }\end{array}$ & Débora Duarte & Isabel C. Fernandes & 041 \\
\hline
\end{tabular}




\begin{tabular}{|c|c|c|c|c|c|}
\hline TCC & ANO & TíTULO & ALUNO & ORIENTADOR & RESUMO \\
\hline 105 & 2006 & $\begin{array}{l}0 \text { trabalho como fator de risco ou proteção para o consumo de } \\
\text { álcool e drogas por mulheres trabalhadoras. }\end{array}$ & Denis R. Galbier da Gama & M. do Perpétuo Socorro de Nóbrega & 042 \\
\hline 106 & 2006 & $\begin{array}{l}\text { Sentimentos e percepções apresentadas por adolescentes durante } \\
\text { a hospitalização. }\end{array}$ & Diana de Menezes Thanasio & Simone de Oliveira Camillo & 043 \\
\hline 107 & 2006 & $\begin{array}{l}\text { Avaliação da situação nutricional das crianças hospitalizadas em } \\
\text { um Hospital Público de Ensino do Grande ABC. }\end{array}$ & Elisa Barbosa Ripar & Rosangela Filipini & 044 \\
\hline 108 & 2006 & $\begin{array}{l}0 \text { significado da vivência do acadêmico trabalhador em enferma- } \\
\text { gem }\end{array}$ & Esther Costa Cutlac & Simone de Oliveira Camillo & 045 \\
\hline 109 & 2006 & Gravidez na adolescência. & Fabrício Camilo Barbosa & Marcelo P. Castellanos & \\
\hline 110 & 2006 & $\begin{array}{l}\text { Implantação do serviço de enfermagem no ambulatório } \\
\text { interdisciplinar de síndrome metabólica e gerontologia da } \\
\text { Faculdade de Medicina do ABC. }\end{array}$ & Fernando H. Britto Bortollotte & Ana Paula Guarnieri & \\
\hline 111 & 2006 & $\begin{array}{l}\text { Parto humanizado: avaliação das práticas utilizadas na assistência } \\
\text { segundo o Ministério da Saúde }\end{array}$ & Flavia Fabiana Pereira & Simone Garcia Lopes & \\
\hline 112 & 2006 & $\begin{array}{l}\text { Estratégias utilizadas pelo trabalhador da enfermagem para lidar } \\
\text { com o estresse laboral. }\end{array}$ & Gina Vilas Boas Lima & Simone de Oliveira Camillo & 046 \\
\hline 113 & 2006 & $\begin{array}{l}\text { As intervenções de enfermagem na admissão de gestantes com } \\
\text { diagnóstico de DHEG. }\end{array}$ & Helena Candido Dimov & Sônia R.G. Lara & \\
\hline 114 & 2006 & $\begin{array}{l}\text { Gravidez na adolescência como fator de risco para o baixo peso } \\
\text { ao nascer }\end{array}$ & Ivanilton Nunes de Almeida & Maria Elisa G. R. Ramos & \\
\hline 115 & 2006 & Influência do sobrepeso na relação sócio-afetiva em adolescentes. & Jaine K. da Silva & Rosangela Filipini & \\
\hline 116 & 2006 & Esquizofrenia: o papel do enfermeiro. & Joceli Moraes da Silva & Sandra T. Amarante & 047 \\
\hline 117 & 2006 & $\begin{array}{l}\text { Freqüência de higiene dos cabelos em pacientes acamados } \\
\text { atendidos em domicílio }\end{array}$ & Juliana Campos Thomaz & Maria Elisa G. R. Ramos & 048 \\
\hline 118 & 2006 & Frequência da episiotomia no parto humanizado & Juliana Ramos de Moraes & Márcia Maciel Rodrigues & 049 \\
\hline 119 & 2006 & $\begin{array}{l}\text { Hábito de lavar as mãos pelos componentes da equipe de enfer- } \\
\text { magem. }\end{array}$ & Luana Duarte de Souza & Loide Corina Chaves & 050 \\
\hline 120 & 2006 & $\begin{array}{l}\text { Indicadores de qualidade da sistematização da assistência de } \\
\text { enfermagem. }\end{array}$ & Maete Diniz & Sandra T. Amarante & 051 \\
\hline 121 & 2006 & Nível de conhecimentos dos enfermeiros sobre a depressão & Mariana Vieira Souza e Silva & M. do Perpétuo Socorro de Nóbrega & 052 \\
\hline 122 & 2006 & A enfermagem e a reprodução humana: uma análise conceitual. & Marta Paula Santos de Sá & Ana Paula Guarnieri & \\
\hline 123 & 2006 & $\begin{array}{l}\text { Sentimentos e percepção apresentados pelo cuidador durante a } \\
\text { hospitalização do adolescente. }\end{array}$ & Michele C. Ribeiro das Chagas & Sônia A. Gonçalves & \\
\hline 124 & 2006 & $\begin{array}{l}\text { Relação entre hábitos alimentares familiares e sobrepeso na infân- } \\
\text { cia. Uma visão da criança. }\end{array}$ & Mônica Jesus da Silva & Rosangela Filipini & \\
\hline 125 & 2006 & Ouvir: um desafio para a assistência de enfermagem. & Natalia Cressoni Theo & Simone de Oliveira Camillo & \\
\hline 126 & 2006 & $\begin{array}{l}\text { Percepção dos usuários de uma unidade básica de saúde em Santo } \\
\text { André sobre planejamento familiar. }\end{array}$ & Odmila Kawahata & $\begin{array}{l}\text { Adozinda de F. Marques Henriques } \\
\text { da Silveira }\end{array}$ & \\
\hline 127 & 2006 & $\begin{array}{l}\text { Sistematização da assistência de enfermagem perioperatória (SAEP) ao } \\
\text { paciente transplantado medular. }\end{array}$ & Patrícia Alves Rodrigues & Maria Elisa G. R. Ramos & 053 \\
\hline 128 & 2006 & $\begin{array}{l}\text { Consultas de enfermagem em saúde coletiva: proposta de um } \\
\text { protocolo. }\end{array}$ & Patrícia M. Rodrigues Belussi & Sandra T. Amarante & 054 \\
\hline 129 & 2006 & $\begin{array}{l}\text { Processo de humanização nas unidades de terapia intensiva: teoria } \\
\text { e prática. }\end{array}$ & Paula Beatriz do Nascimento & Márcia Maciel Rodrigues & 055 \\
\hline 130 & 2006 & $\begin{array}{l}\text { Contracepção feminina: a colaboração do parceiro no controle de } \\
\text { natalidade sobre a perspectiva da mulher. }\end{array}$ & Priscila Ruiz Alves & Sônia R.G. Lara & 056 \\
\hline 131 & 2006 & $\begin{array}{l}\text { A percepção dos enfermeiros acerca das necessidades sócio- } \\
\text { afetivas do paciente hospitalizado }\end{array}$ & Raquel Izolani & Ana Maria Fiorano & 057 \\
\hline 132 & 2006 & $\begin{array}{l}\text { Perfil dos hipertensos que não aderem ao tratamento da } \\
\text { hipertensão na unidade de saúde do Jardim Irene. }\end{array}$ & $\begin{array}{l}\text { Rosana de Fátima Queiroz de Luna } \\
\text { Gomes }\end{array}$ & Loide Corina Chaves & 058 \\
\hline 133 & 2006 & $\begin{array}{l}\text { Fatores que determinam complicações ao recém-nascidos: uma } \\
\text { contribuição ao planejamento de enfermagem. }\end{array}$ & Silvia Ferreira & Isabel C. Fernandes & \\
\hline 134 & 2006 & $\begin{array}{l}\text { Uso e abuso de drogas licitas e ilícitas por graduandos de enfer- } \\
\text { magem. }\end{array}$ & Thiago Sterling Wochnik & M. do Perpétuo Socorro de Nóbrega & 059 \\
\hline 135 & 2006 & $\begin{array}{l}\text { Aderência da equipe de enfermagem, referente às medidas orienta- } \\
\text { doras da CCIH para redução do índice de infecção hospitalar. }\end{array}$ & Tiziana Roberta Zoffoli & Loide Corina Chaves & 060 \\
\hline 136 & 2006 & $\begin{array}{l}\text { Percepção do aluno de graduação em enfermagem sobre brinque- } \\
\text { doteca hospitalar }\end{array}$ & Tuany Spada de Pastena & Sônia A. Gonçalves & \\
\hline 137 & 2006 & $\begin{array}{l}\text { Auditoria da sistematização da assistência de enfermagem em uma } \\
\text { unidade de terapia intensiva. }\end{array}$ & Valeria Sigolo Gracio & Sandra T. Amarante & 061 \\
\hline 138 & 2007 & $\begin{array}{l}0 \text { conhecimento de crianças, vivendo em condições sócio- } \\
\text { econômicas desfavoráveis, sobre hábitos de higiene e preservação } \\
\text { do meio ambiente. }\end{array}$ & Ailita D'Arc Veronica Miguel & Rosangela Filipini & 062 \\
\hline 139 & 2007 & $\begin{array}{l}\text { Nível de dor experimentado por puérperas diante das alterações } \\
\text { mamilares decorrentes do período de lactação. }\end{array}$ & Ana Carolina Siqueira & Sônia A. Gonçalves & 063 \\
\hline
\end{tabular}




\begin{tabular}{|c|c|c|c|c|c|}
\hline TCC & ANO & TíTULO & ALUNO & ORIENTADOR & RESUMO \\
\hline 140 & 2007 & $\begin{array}{l}\text { Avaliação dos níveis pressóricos dos indivíduos atendidos no ambu- } \\
\text { latório de cardiologia da FMABC. }\end{array}$ & Andressa Grace Ferrara Andre & Maria Elisa G. R. Ramos & 064 \\
\hline 141 & 2007 & Consumo de álcool, tabaco e drogas ilícitas durante a gravidez. & Camila Guilherme Martini & M. do Perpétuo Socorro de Nóbrega & 065 \\
\hline 142 & 2007 & $\begin{array}{l}\text { Identificação de sintomas depressivos em profissionais de enferma- } \\
\text { gem que atuam nas emergências psiquiátricas. }\end{array}$ & Carla Portela da Silva & Simone de Oliveira Camillo & 066 \\
\hline 143 & 2007 & $\begin{array}{l}\text { Fenômenos depressivos na doença cardiovascular: identificação } \\
\text { precoce para prevenção de complicações. }\end{array}$ & Cristiane Wenzel & Ana Maria Fiorano & 067 \\
\hline 144 & 2007 & $\begin{array}{l}\text { Imunização de recém-nascidos prematuros de baixo peso ao } \\
\text { nascer. }\end{array}$ & Debora Cristina Silva & Sônia A. Gonçalves & \\
\hline 145 & 2007 & Hemodiálise e o cuidar. & Debora Francisco & Sandra T. Amarante & 068 \\
\hline 146 & 2007 & $\begin{array}{l}\text { Validação do processo de esterilização de materiais embalados em } \\
\text { papel crepado. }\end{array}$ & Edilania de Alencar Franca & Maria Elisa G. R. Ramos & 069 \\
\hline 147 & 2007 & $\begin{array}{l}\text { Frequência de gestantes portadoras de diabetes gestacional no } \\
\text { Centro de Saúde-Escola no município de Santo André. }\end{array}$ & Elena da Silva Ferreira & Tânia Albino & 070 \\
\hline 148 & 2007 & $\begin{array}{l}\text { Cuidados paliativos: interação entre a equipe multidisciplinar e a familia } \\
\text { de paciente oncológico terminal. }\end{array}$ & Fabiola de Almeida Godoi & Márcia Maciel Rodrigues & 071 \\
\hline 149 & 2007 & $\begin{array}{l}\text { Avaliação do auto-conceito em crianças obesas na faixa etária de } 6 \\
\text { a } 10 \text { anos em um ambiente escolar. }\end{array}$ & Gabriela Pinheiro Bafa & Rosangela Filipini & 072 \\
\hline 150 & 2007 & $\begin{array}{l}\text { A família durante o processo de hospitalização da criança e do } \\
\text { adolescente. }\end{array}$ & Gislene Francine da Silva & Isabel C. Fernandes & 073 \\
\hline 151 & 2007 & $\begin{array}{l}\text { Frequência de gestantes portadoras de doença hipertensiva em } \\
\text { Centro de Saúde no município de Santo André. }\end{array}$ & Gleice Afonso Xavier Franco & Sônia A. Gonçalves & \\
\hline 152 & 2007 & $\begin{array}{l}\text { Avaliação do desempenho da equipe de enfermagem gente ao } \\
\text { procedimento da punção venosa periférica. }\end{array}$ & Josiane Pereira da Silva & Loide Corina Chaves & 074 \\
\hline 153 & 2007 & $\begin{array}{l}\text { Stress da equipe de enfermagem no ambiente cirúrgico de um } \\
\text { Hospital particular do ABC. }\end{array}$ & Juciara Ribeiro de Oliveira & Maria Elisa G. R. Ramos & \\
\hline 154 & 2007 & $\begin{array}{l}\text { Conhecimento das mães sobre } 0 \text { aleitamento materno em uma } \\
\text { unidade neonatal. }\end{array}$ & Juliane Regina Camargo & Isabel C. Fernandes & 075 \\
\hline 155 & 2007 & $\begin{array}{l}\text { Depressão em mães com filhos oncológicos em tratamento } \\
\text { quimioterápico. }\end{array}$ & Karina Bartalini & Simone de Oliveira Camillo & 076 \\
\hline 156 & 2007 & $\begin{array}{l}\text { Percepções e sentimentos dos profissionais de enfermagem em } \\
\text { relação ao paciente com transtorno mental }\end{array}$ & Keli C. A. dos Santos & Simone de Oliveira Camillo & 077 \\
\hline 157 & 2007 & $\begin{array}{l}0 \text { trabalho da equipe multidisciplinar frente ao processo de qualidade } \\
\text { implantada na Instituição Hospitalar. }\end{array}$ & Larissa de Siqueira Gutierres & Márcia Maciel Rodrigues & 078 \\
\hline 158 & 2007 & Fatores que interferem na dieta adequada do idoso. & Lidiane dos Santos Barbosa & Ana Paula Guarnieri & \\
\hline 159 & 2007 & $\begin{array}{l}\text { Fatores determinantes para adesão do pré-natal entre mulheres } \\
\text { atendidas em Capuava- Santo André. }\end{array}$ & Maria Ap. Paranhos de Oliveira & Magali Motta & \\
\hline 160 & 2007 & $\begin{array}{l}\text { Proposta de projeto de posto de captação de doadores de medula } \\
\text { óssea. }\end{array}$ & $\begin{array}{l}\text { Maria Aparecida Lopes Santos } \\
\text { Fracaroli }\end{array}$ & Maria Belén Salazar Posso & 079 \\
\hline 161 & 2007 & $\begin{array}{l}\text { latrogenias no processo de trabalho da equipe de enfermagem: } \\
\text { estratégias de enfrentamento pelo enfermeiro. }\end{array}$ & Mariana de Oliveira Tonini Milanez & Sandra T. Amarante & 080 \\
\hline 162 & 2007 & $\begin{array}{l}0 \text { enfrentamento da doença neoplásica por crianças hospitalizadas } \\
\text { e em tratamento ambulatorial, segundo avaliação de ansiedade. }\end{array}$ & Natalia Cristina Liubartas & Rosangela Filipini & 081 \\
\hline 163 & 2007 & $\begin{array}{l}\text { Frequência de sintomas depressivos em idosos cadastrados no } \\
\text { Projeto Bem Viver. }\end{array}$ & Natalie Duarte Carbonin & Ana Paula Guarnieri & \\
\hline 164 & 2007 & $\begin{array}{l}\text { Caracterização de mulheres atendidas na Casa da Gestante de Alto } \\
\text { Risco de São Bernardo do Campo no período de janeiro a junho de } \\
2006 \text {. }\end{array}$ & Noely Cristina Teixeira & Rosangela Filipini & 082 \\
\hline 165 & 2007 & $\begin{array}{l}\text { Níveis de estresse e consumo de álcool em trabalhadores de } \\
\text { transporte coletivo. }\end{array}$ & Odirley Kawahata & M. do Perpétuo Socorro de Nóbrega & 083 \\
\hline 166 & 2007 & $\begin{array}{l}\text { Qualidade de vida dos estudantes trabalhadores do curso de } \\
\text { graduação em Enfermagem. }\end{array}$ & Patricia Neiva da Silva & Ana Maria Fiorano & \\
\hline 167 & 2007 & $\begin{array}{l}\text { Sentimentos e percepções dos adolescentes frente ao câncer e a } \\
\text { quimioterapia. }\end{array}$ & Patrícia Thessotto Vaz Fernandes & Simone de Oliveira Camillo & 084 \\
\hline 168 & 2007 & Impacto negativo que o álcool causa no ambiente familiar. & Rafaela Zeferino de Lima & M. do Perpétuo Socorro de Nóbrega & \\
\hline 169 & 2007 & $\begin{array}{l}\text { Teste do pezinho: } 0 \text { conhecimento das mães quanto a sua } \\
\text { importância. }\end{array}$ & Sheila Tatiana Macedo Borim & Sônia A. Gonçalves & 085 \\
\hline 170 & 2007 & $\begin{array}{l}\text { Cirurgia Barítrica em um hospital de ensino da região do ABC de } \\
2004 \text { a } 2006 \text {. }\end{array}$ & Simone Aparecida Savella & Loide Corina Chaves & 086 \\
\hline 171 & 2007 & $\begin{array}{l}\text { Projeto piloto: a assistência de enfermagem no apoio aos } \\
\text { cuidadores familiares de pacientes acometidos por processos } \\
\text { demenciais }\end{array}$ & Suelen Augusto de Oliveira & Ana Paula Guarnieri & 087 \\
\hline 172 & 2007 & $\begin{array}{l}\text { Concepções sobre sexualidade e acesso a informações pelo } \\
\text { adolescente. }\end{array}$ & Tatiana Martins d'Avila & Érica C. Araújo & 088 \\
\hline 173 & 2007 & $\begin{array}{l}\text { Qualidade de vida: estratégias de ação da equipe de enfermagem } \\
\text { em atividades físicas e laborais na senescência }\end{array}$ & Tauny de Souza & Sandra T. Amarante & 089 \\
\hline
\end{tabular}




\begin{tabular}{|c|c|c|c|c|c|}
\hline TCC & ANO & TíTULO & ALUNO & ORIENTADOR & RESUMO \\
\hline 174 & 2007 & Pressão arterial: técnica de aferição e variações de resultados. & Telma do Nascimento & Ana Maria Fiorano & 090 \\
\hline 175 & 2007 & Processo de cuidados de enfermagem com úlceras por pressão. & Thais Naiene Piveta & Sandra T. Amarante & \\
\hline 176 & 2007 & $\begin{array}{l}\text { Implementação do processo da sistematização da assistência de } \\
\text { enfermagem no pré-operatório de cirurgia eletiva do idoso. }\end{array}$ & Vera Lúcia Maria Alves de Melo & Márcia Maciel Rodrigues & \\
\hline 177 & 2008 & $\begin{array}{l}\text { Processo de acolhimento dos acompanhantes de doentes instituci- } \\
\text { onalizados na UTI adulto }\end{array}$ & Aline Alves de Souza & Márcia Maciel Rodrigues & \\
\hline 178 & 2008 & $\begin{array}{l}\text { Dificuldades dos enfermeiros quanto à aplicação e interpretação da } \\
\text { Escala de Coma de Glagow }\end{array}$ & Amanda Gonçalves Carrico & Ana Maria Fiorano & 091 \\
\hline 179 & 2008 & $\begin{array}{l}0 \text { cinema como recurso pedagógico na disciplina de enfermagem } \\
\text { terapêutica }\end{array}$ & Amanda Regina da Silva Nicolau & Simone de Oliveira Camillo & \\
\hline 180 & 2008 & A inserção da mulher negra na enfermagem brasileira & Amanda Ricci & Maria Belén Salazar Posso & 092 \\
\hline 181 & 2008 & $\begin{array}{l}\text { Visão dos cuidadores atendidos no ambulatório de distúrbio da } \\
\text { memória, quanto ao fornecimento da medicação de alto custo pelo } \\
\text { governo. }\end{array}$ & $\begin{array}{l}\text { Ângela Cristina de Oliveira e Tânia } \\
\text { Regina Martins }\end{array}$ & Márcia Maciel Rodrigues & 093 \\
\hline 182 & 2008 & Atendimento pré-hospitalar e a existência de não conformidades. & Bruna Mazine & Márcia Maciel Rodrigues & \\
\hline 183 & 2008 & $\begin{array}{l}\text { Infecção ocupacional pelo vírus da hepatite B: riscos e medidas de } \\
\text { prevenção. }\end{array}$ & Bruno Francisco Real de Lima & Loide Corina Chaves & 094 \\
\hline 184 & 2008 & $\begin{array}{l}\text { Proposta de elaboração de um manual de orientações para } \\
\text { pacientes inseridos nos serviços de Reprodução Humana Assistida } \\
\text { da FMABC. }\end{array}$ & $\begin{array}{l}\text { Camila Ap. Santos e Caroline Miraldo } \\
\text { Lopes }\end{array}$ & Maria Elisa G. R. Ramos & \\
\hline 185 & 2008 & A SAE no idoso institucionalizado. & $\begin{array}{l}\text { Cristina Melo R. Tavares da Silva e } \\
\text { Rebeca Paschoalato da Costa }\end{array}$ & Sandra T. Amarante & \\
\hline 186 & 2008 & A aderência medicamentosa de idosos no Projeto Bem Viver & Daniela Adriano Ferreira & Ana Paula Guarnieri & \\
\hline 187 & 2008 & $\begin{array}{l}\text { Caracterização das gestantes atendidas no pré-natal de alto risco } \\
\text { no CAISM-SBC. }\end{array}$ & $\begin{array}{l}\text { Daniela Fernanda Alves e Rafaela } \\
\text { Casadei Medugno }\end{array}$ & Tânia Albino & 095 \\
\hline 188 & 2008 & $\begin{array}{l}\text { Impacto do cuidar: a visão dos cuidadores de indivíduos portadores } \\
\text { de distúrbios de memória. }\end{array}$ & $\begin{array}{l}\text { Daniella Almeida de Oliveira e Sandra } \\
\text { Piorello Viana }\end{array}$ & M. do Perpétuo Socorro de Nóbrega & 096 \\
\hline 189 & 2008 & $\begin{array}{l}\text { Nível de informações das orientações de enfermagem a gestante de } \\
\text { baixo risco no CSE- Capuava. }\end{array}$ & $\begin{array}{l}\text { Elaine Marta de Lima e Michelle } \\
\text { Domingos Souza }\end{array}$ & Magali Motta & \\
\hline 190 & 2008 & Cuidados paliativos aos pacientes terminais & Emilene T. dos S. Souza & Sandra T. Amarante & \\
\hline 191 & 2008 & $\begin{array}{l}\text { Plano de alta de enfermagem ao paciente submetido à revasculari- } \\
\text { zação do miocárdio. }\end{array}$ & $\begin{array}{l}\text { Flávia Araújo Pitta e Vanessa C. } \\
\text { Araújo Di Stefano }\end{array}$ & Ana Maria Fiorano & 097 \\
\hline 192 & 2008 & $\begin{array}{l}\text { Proposta de um instrumento para avaliação de risco de moradia } \\
\text { para idosos }\end{array}$ & Gabriela Giubilato & $\begin{array}{l}\text { Simone Garcia Lopes e Ana Paula } \\
\text { Guarnieri }\end{array}$ & 098 \\
\hline 193 & 2008 & Assistência de enfermagem ao paciente grande queimado na UTI & Isabela Delboni & Ana Maria Fiorano & 099 \\
\hline 194 & 2008 & 0 universo do aluno do $4^{\circ}$ ano de graduação em enfermagem & $\begin{array}{l}\text { Isis Harumi Muraki e Nívea Tsuiami } \\
\text { Sato Kazama }\end{array}$ & Simone de Oliveira Camillo & \\
\hline 195 & 2008 & Avaliação da dor em recém-nascidos submetidos a punção venosa. & Izildinha de Jesus & Sônia A. Gonçalves & 100 \\
\hline 196 & 2008 & A importância de ouvir na assistência de enfermagem & Juliane Garcia da Silva & Simone de Oliveira Camillo & \\
\hline 197 & 2008 & $\begin{array}{l}\text { Frequência da prematuridade relacionada a doença hipertensiva } \\
\text { especifica da gravidez }\end{array}$ & Karen C. da Silveira Rosa & Tânia Albino & 101 \\
\hline 198 & 2008 & Materiais reesterilizados: suas causas específicas. & Lívia Penha Ferraro & Maria Elisa G. R. Ramos & 102 \\
\hline 199 & 2008 & Estresse do cuidador de crianças hospitalizadas. & Marina Macedo daminato & Sandra T. Amarante & \\
\hline 200 & 2008 & Fatores que favorecem a prática do aleitamento materno & Mirleine Colman S. de Carvalho & Sônia A. Gonçalves & 103 \\
\hline 201 & 2008 & $\begin{array}{l}\text { A percepção dos graduandos de enfermagem em relação ao } \\
\text { cuidado com o paciente usuário crônico de álcool. }\end{array}$ & $\begin{array}{l}\text { Patrícia da Silveira João e Veronica } \\
\text { Fontanillas Souza }\end{array}$ & Simone Oliveira Camillo & 104 \\
\hline 202 & 2008 & $\begin{array}{l}\text { A presença materna na saída da sala de recuperação pós-anestési- } \\
\text { ca em crianças de zero a doze anos. }\end{array}$ & Patrícia Herrera Dias & Maria Elisa G. R. Ramos & 105 \\
\hline 203 & 2008 & $\begin{array}{l}\text { A abordagem da saúde na população negra na Faculdade de } \\
\text { Medicina do ABC }\end{array}$ & Rebeca Aletheia R. Santana & $\begin{array}{l}\text { Marcelo P. Castellanos e Adozinda } \\
\text { M. Silveira }\end{array}$ & \\
\hline 204 & 2008 & $\begin{array}{l}\text { Implicações da displasia bronco pulmonar para a assistência de } \\
\text { enfermagem. }\end{array}$ & Renata Colamarche & Isabel C. Fernandes & 106 \\
\hline 205 & 2008 & $\begin{array}{l}\text { Avaliação dos efeitos da homeopatia como terapia coadjuvante em } \\
\text { crianças com câncer. }\end{array}$ & Thaís Morales Carraro & Rosangela Filipini & 107 \\
\hline
\end{tabular}

